\title{
Application of Mathematical and Three- Dimensional Computer Modeling Tools in the Planning of Processes of Fuel and Energy Complexes
}

\author{
Olesya Aksenova ${ }^{1 *}$, Evgenia Nikolaeva $^{1}$, and Michal Cehlár ${ }^{2}$ \\ ${ }^{1}$ T.F. Gorbachev Kuzbass State Technical University, 650000, 28 Vesennyaya St., Kemerovo, Russia \\ ${ }^{2}$ Technical University of Kosice, Faculty of Mining, Ecology, Process Control and Geotechnologies, \\ Letná 9, 04200 Kosice, Slovak republic
}

\begin{abstract}
This work aims to investigate the effectiveness of mathematical and three-dimensional computer modeling tools in the planning of processes of fuel and energy complexes at the planning and design phase of a thermal power plant (TPP). A solution for purification of gas emissions at the design development phase of waste treatment systems is proposed employing mathematical and three-dimensional computer modeling - using the E-nets apparatus and the development of a 3D model of the future gas emission purification system. Which allows to visualize the designed result, to select and scientifically prove economically feasible technology, as well as to ensure the high environmental and social effect of the developed waste treatment system. The authors present results of a treatment of planned technological processes and the system for purifying gas emissions in terms of E-nets, using mathematical modeling in the Simulink application. What allowed to create a model of a device from the library of standard blocks and to perform calculations. A three-dimensional model of a system for purifying gas emissions has been constructed. It allows to visualize technological processes and compare them with the theoretical calculations at the design phase of a TPP and, if necessary, make adjustments.
\end{abstract}

\section{Introduction}

At the present day, the fuel industry is the basis for the development of the Russian economy, an instrument for carrying out domestic and foreign policy. An accurate modeling processes of the fuel and energy complex is considered to be very important. In addition, the operation of such facilities due to the products of coal combustion processing entails massive emissions of gases into the environment. Thus, violating the ecological and industrial safety of the world around us. Therefore, when planning and modeling processes of fuel and energy complexes, it is necessary to take into account the availability of waste treatment systems. Namely a system for purifying gas emissions and a system for the full cycle

\footnotetext{
*Corresponding author: olesya_aksenova42@mail.ru
} 
of processing ash and slag wastes that will not only ensure environmental and industrial safety, but will also be cost-effective for enterprises and the country as a whole.

\section{Materials and Methods}

At the stage of planning the processes of fuel and energy complexes and the development of technical documentation, a modern range of computer programs allows to present an operation analysis of a future TPP from different perspectives. Mathematical modeling [9] makes it possible to verify the model correctness and adjust it at the stage of construction, while 3D computer modeling allows to obtain a realistic image based on available technical documentation, identify technical errors, and correct, during the planning stage, individual technological processes and a TPP as a whole.

When performing a mathematical analysis, it is advisable to use the E-nets apparatus [2]. As an extension of Petri nets, E-nets are a graphical and mathematical tool used to model systems of different types.

An abstracted model of a TPP, shown in Fig. 1, with the possibility of expansion is considered in this work.

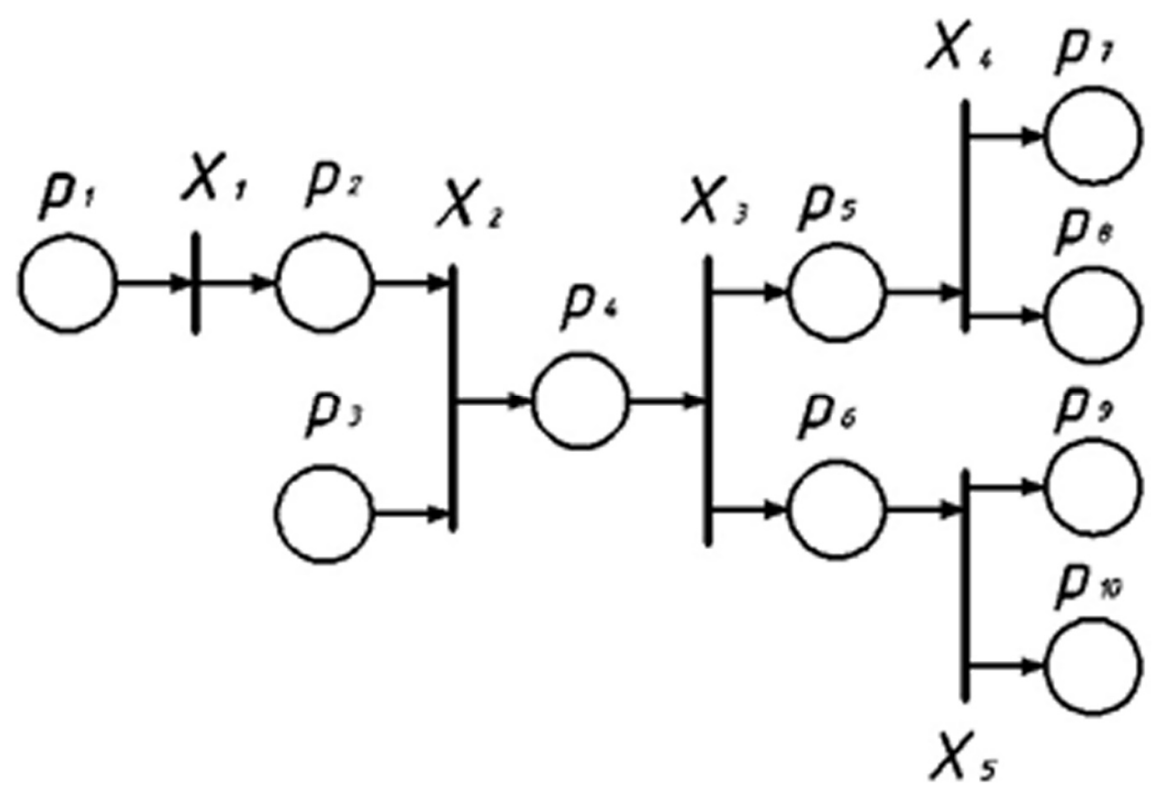

Fig. 1. A TPP model in terms of E-nets.

Where $\mathrm{p}_{1}$ - a new portion of coal entered a crushing plant; $\mathrm{p}_{2}$ - coal is processed; $\mathrm{p}_{3}$ - condensate and replenished water losses entered the storage facility; $\mathrm{p}_{4}$ - the raw material was supplied to the combustion chamber; $\mathrm{p}_{5}$ - combustion products are obtained; $\mathrm{p}_{6}-$ steam is fed to the turbine; $p_{7}$ - has and slag waste are transferred to the processing system; $p_{8}$ gas-like wastes enter the purification system; $p_{9}$ - electric power is allocated to external consumers; $p_{10}$ - steam from the turbine selections goes to a TPP; $X_{1}=\left(T, 0, \varphi_{1}\right)$ - coal processing; $\varphi_{1}=\mathrm{L}\left(\mathrm{X}_{1}\right)=\left\{\mathrm{p}_{1} \mathrm{X}_{1} \mid \mathrm{X}_{1} \rightarrow \mathrm{p}_{1} \mathrm{p}_{2}\right\}=\left\{\mathrm{p}_{1} \mathrm{p}_{2}\right\}, \mathrm{X}_{2}=\left(\mathrm{I}, 0, \varphi_{2}\right)$ - loading of raw materials into the combustion chamber; $\varphi_{2}=\mathrm{L}\left(\mathrm{X}_{2}\right)=\left\{\mathrm{p}_{2} \mathrm{p}_{3} \mathrm{X}_{2} \mid \mathrm{X}_{2} \rightarrow \mathrm{p}_{2} \mathrm{p}_{3} \mathrm{p}_{4}\right\}=>$ $L\left(\mathrm{X}_{2}\right)=\left\{\mathrm{p}_{2} \mathrm{p}_{3} \mathrm{p}_{4}\right\}, \quad \mathrm{X}_{3}=\left(\mathrm{F}, 0, \varphi_{3}\right) \quad$ raw materials processing; $\varphi_{3}=\mathrm{L}\left(\mathrm{X}_{3}\right)=$ 
$\left\{\mathrm{p}_{4} \mathrm{X}_{3} \mid \mathrm{X}_{3} \rightarrow \mathrm{p}_{4} \mathrm{p}_{5} \mathrm{p}_{6}\right\}=\left\{\mathrm{p}_{4} \mathrm{p}_{5} \mathrm{p}_{6}\right\}, \quad \mathrm{X}_{4}=\left(\mathrm{F}, 0, \varphi_{4}\right) \quad$ - waste disposal; $\varphi_{4}=\mathrm{L}\left(\mathrm{X}_{4}\right)=$ $\left\{\mathrm{p}_{5} \mathrm{X}_{4} \mid \mathrm{X}_{4} \rightarrow \mathrm{p}_{5} \mathrm{p}_{7} \mathrm{p}_{8}\right\}=\left\{\mathrm{p}_{5} \mathrm{p}_{7} \mathrm{p}_{8}\right\}, \mathrm{X}_{5}=\left(\mathrm{F}, 0, \varphi_{5}\right)$ - power generation and steam extraction; $\varphi_{5}=\mathrm{L}\left(\mathrm{X}_{5}\right)=\left\{\mathrm{p}_{6} \mathrm{X}_{5} \mid \mathrm{X}_{5} \rightarrow \mathrm{p}_{6} \mathrm{p}_{9} \mathrm{p}_{10}\right\}=\left\{\mathrm{p}_{6} \mathrm{p}_{9} \mathrm{p}_{10}\right\}$, the initial marking is represented by the vector: $(1,0,1,0,0,0,0,0,0,0)$.

Software packages for numerical computations MatLab [1,3] and Simulink [4] were used to implement the developed E-nets [5] model on a computer.

\section{Results and discussion}

Modeling using Simulink implements the principle of visual programming, according to which, the user create a model of a device on a screen using the library of standard blocks and performs calculations. At the same time, in contrast to classical ways of modeling, the user does not need to thoroughly study the programming language and numerical methods. Basic computer working skills and, of course, knowledge of the subject area are sufficient.

The model created in Simulink, shown in Fig. 2, is a balance model of material flows [6], in which the following technological phases were realized:

1. The loss of coal in its preparation for burning,

2. Electric power generation,

3. Formation and processing of ash and slag wastes,

4. Formation and purification of gas emissions.

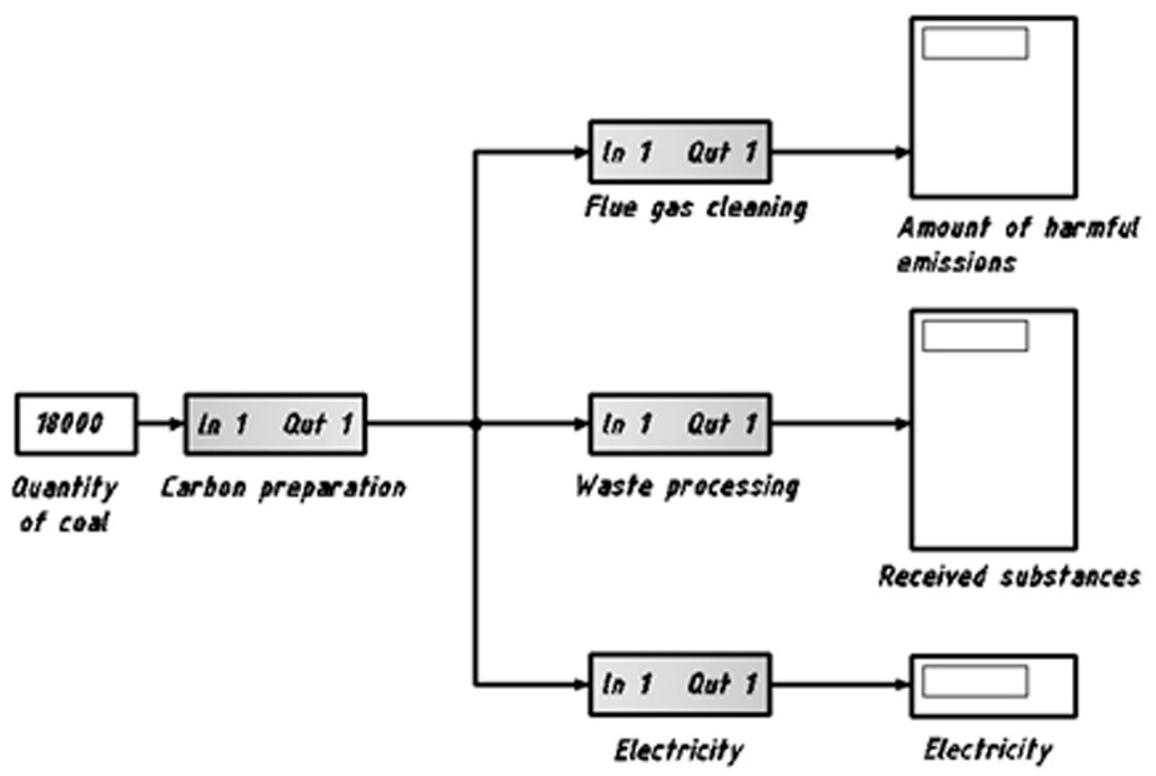

Fig. 2. The balance model of material flows of a TPP.

In order to make the model more understandable and easier to expand, the processing and purification blocks were implemented as subsystems. Consider a subsystem for purification of gas emissions of a TPP, which in turn also consists of several modules.

The first in the sequence of these modules is an electrostatic precipitator. The following formula was used for its efficiency calculation:

$$
\mathrm{M}_{\mathrm{T}}=\mathrm{A} \cdot \mathrm{m} \cdot \chi \cdot\left(1-\frac{\eta_{\tau}}{100}\right),
$$


where $\mathrm{M}_{\mathrm{T}}$ - is the gross emission of solid particles in flue gases; $\mathrm{A}$ is the ash content of fuel, in $\% ; \mathrm{m}$ is the amount of fuel consumed per year, ton; $\chi$ is a dimensionless coefficient characterizing the fraction of ash carried away with flue gases, depends on furnace and fuel types; $\eta_{\tau}$ is the efficiency of ash collectors, in $\%$.

The next module is a system for purification from nitrogen oxides. To determine its characteristics, the following formula was used:

$$
\mathrm{M}_{\mathrm{NO}_{2}}=\mathrm{m} \cdot \mathrm{Q}^{\mathrm{H}} \cdot \mathrm{K}_{\mathrm{NO}_{2}} \cdot(1-\beta) \cdot 10^{-3},
$$

where $\mathrm{M}_{\mathrm{NO}_{2}}$ is the gross release of nitrogen oxide in terms of nitrogen dioxide; $\mathrm{Q}^{\mathrm{H}}$ is the lowest heat of combustion of natural fuel, $\mathrm{MJ} / \mathrm{kg} ; \mathrm{K}_{\mathrm{NO}_{2}}$ is a parameter characterizing the amount of nitrogen oxides generated per GJ of heat, $\mathrm{kg} / \mathrm{GJ} ; \beta$ is a coefficient which depends on the degree of reduction of nitrogen oxide emissions resulting from the application of technical solutions.

Desulphurization system. When calculating the performance of this module, the following formula was used:

$$
\mathrm{M}_{\mathrm{SO}_{2}}=0,02 \cdot \mathrm{m} \cdot \mathrm{S} \cdot\left(1-\eta_{\mathrm{SO}_{2}}^{1}\right) \cdot\left(1-\eta_{\mathrm{SO}_{2}}^{2}\right),
$$

where $\mathrm{M}_{\mathrm{SO}_{2}}$ is the total emission of sulfur oxides in terms of sulfur dioxide; $\mathrm{S}$ is the sulfur content, in \%; $\eta_{\mathrm{SO}_{2}}^{1}$ is the proportion of sulfur oxides bound by the flying ash of the fuel; $\eta_{\mathrm{SO}_{2}}^{2}$ is the proportion of sulfur oxides trapped in the ash collector.

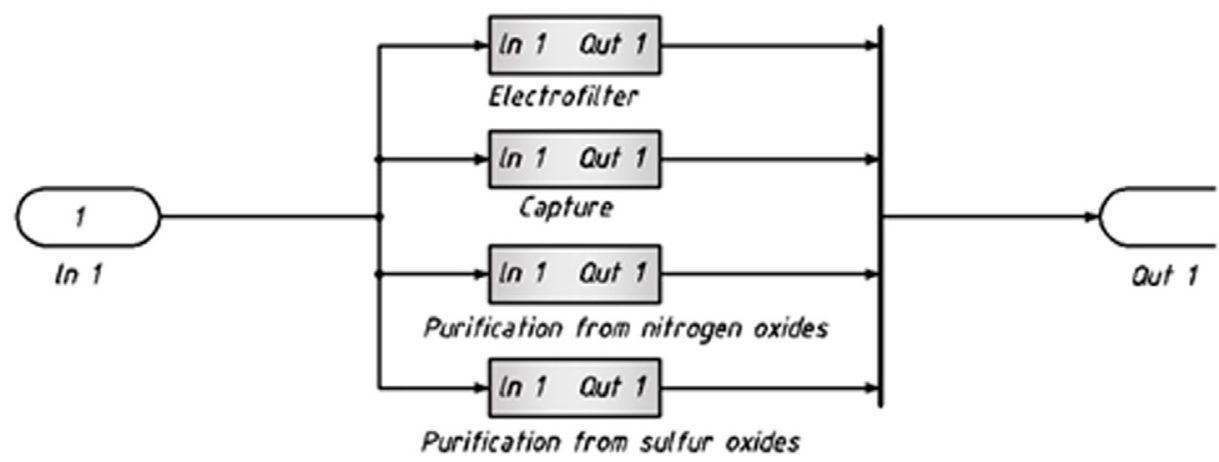

Fig. 3. The subsystem purifying gas emissions.

As an example of mathematical and three-dimensional computer modeling, we consider in more detail the subsystem of carbon dioxide capture.

Carbon dioxide is one of the greenhouse gases, the excess of which in the atmosphere leads to global climate change. Throughout the world, numerous attempts are being made to reduce greenhouse gas emissions and one of these methods - capture and storage of carbon dioxide (Carbon Capture \& Storage - CCS) - is becoming increasingly widespread.

The system of carbon dioxide capture and storage is divided into three stages: capture, transportation, and storage. Consider the first stage shown in Fig. 4. 


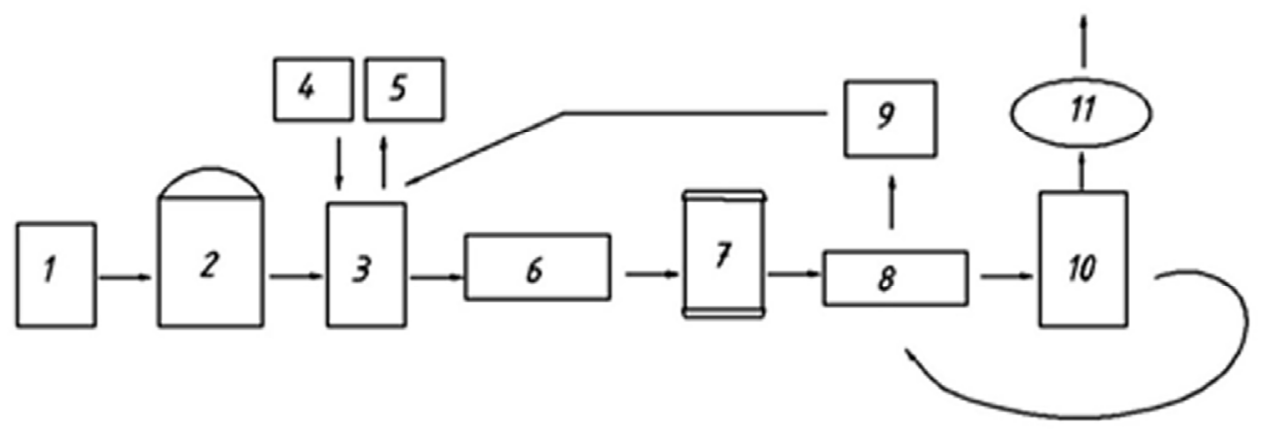

Fig. 4. Flow diagram of the carbon dioxide capture process.

The technology of carbon dioxide capture and concentration is realized according to the following scheme: the flue gases (1) are fed by the induced draft fan to the centrifugal apparatus (2) to remove suspended particles and reduce the temperature of the gas stream by passing the gas mixture through the water layer. The cooled gas stream is fed to the packed absorber (3), which also receives the regenerated absorbent solution (4). The gas purified in the absorber is extracted from the unit, and the solution saturated with an acid gas is removed from the built into the absorber vessel by the pump (6) through the filter (7) and the recuperative heat exchanger (8) and fed to regenerate into the packed desorber (10) heated from below by the heaters. The acid gas from the top of the desorber is fed into the compressor (11), then it is removed from the apparatus. The regenerated solution from the bottom of the desorber is moved through the recuperative heat exchanger (8) and the refrigerator (9) into the absorber for further operation.

The model of the carbon dioxide capture and storage system in terms of E-nets has the following form (Fig. 5): $p_{1}$ - flue gases entered the centrifugal apparatus; $p_{2}$ - a cooled gas is fed to a packed absorber; $p_{3}$ - the gas purified in the absorber is removed from the unit; $\mathrm{p}_{4}$ - the solution saturated with the acid gas passes through the filter; $\mathrm{p}_{5}$ - the solution passes through the recuperative heat exchanger; $\mathrm{p}_{6}$ - the solution is recovered in a packed desorber; $\mathrm{p}_{7}$ - the acid gas at the top of the desorber is removed from the unit; $\mathrm{p}_{8}$ - the solution at the bottom of the desorber is regenerated; $p_{9}$ - the regenerated solution enters the refrigerator.

$\mathrm{X}_{1}=\left(\mathrm{Y}, 0, \varphi_{1}\right)-$ process of regeneration in desorber;

$$
\begin{aligned}
& \varphi_{1}=L\left(X_{1}\right)=\left\{\begin{array}{l}
p_{1} \rightarrow X_{1}, p_{9} \rightarrow X_{1}, r_{1} \rightarrow X_{1}, X_{1} \rightarrow p_{2} \leq>r_{1}=0 \vee \\
p_{1} \rightarrow X_{1}, p_{9} \rightarrow X_{1}, r_{1} \rightarrow X_{1}, X_{1} \rightarrow p_{2}<=>r_{1}=1 \vee
\end{array}\right. \\
& \left.\begin{array}{l}
\vee\left(p_{1}=1, p_{9}=0, p_{2}=0,\right) \rightarrow p_{1} p_{2} \\
V\left(p_{1}=1, p_{9}=1, p_{2}=0,\right) \rightarrow p_{1} p_{9} p_{2}
\end{array}\right\}=>L\left(X_{1}\right)=\left\{p_{1} p_{2} \wedge p_{1} p_{9} p_{2}\right\}
\end{aligned}
$$

$X_{2}=\left(F, 0, \varphi_{2}\right)-$ the solution is fed for regeneration into the desorber;

$$
\varphi_{2}=\mathrm{L}\left(\mathrm{X}_{2}\right)=\left\{\mathrm{p}_{2} \mathrm{X}_{2} \mid \mathrm{X}_{2} \rightarrow \mathrm{p}_{2} \mathrm{p}_{3} \mathrm{p}_{4}\right\}=\left\{\mathrm{p}_{2} \mathrm{p}_{3} \mathrm{p}_{4}\right\},
$$

$\mathrm{X}_{3}=\left(\mathrm{Y}, 0, \varphi_{3}\right)-$ the solution is fed for regeneration into the desorber;

$\varphi_{3}=L\left(X_{3}\right)=\left\{\begin{array}{l}p_{4} \rightarrow X_{3}, p_{8} \rightarrow X_{3}, r_{2} \rightarrow X_{3}, X_{3} \rightarrow p_{5} \leq>r_{2}=0 \vee \\ p_{4} \rightarrow X_{3}, p_{8} \rightarrow X_{3}, r_{2} \rightarrow X_{3}, X_{3} \rightarrow p_{5}<=>r_{2}=1 \vee\end{array}\right.$

$\left.\begin{array}{c}\vee\left(p_{4}=1, p_{8}=0, p_{5}=0,\right) \rightarrow p_{4} p_{5} \\ \vee\left(p_{4}=1, p_{8}=1, p_{5}=0,\right) \rightarrow p_{4} p_{8} p_{5}\end{array}\right\}=>L\left(X_{3}\right)=\left\{p_{4} p_{5} \wedge p_{4} p_{8} p_{5}\right\}$

$\mathrm{X}_{4}=\left(\mathrm{F}, 0, \varphi_{4}\right)-$ the solution is fed for regeneration into the desorber;

$$
\varphi_{4}=\mathrm{L}\left(\mathrm{X}_{4}\right)=\left\{\mathrm{p}_{5} \mathrm{X}_{4} \mid \mathrm{X}_{4} \rightarrow \mathrm{p}_{5} \mathrm{p}_{6} \mathrm{p}_{9}\right\}=\left\{\mathrm{p}_{5} \mathrm{p}_{6} \mathrm{p}_{9}\right\},
$$

$\mathrm{X}_{5}=\left(\mathrm{F}, 0, \varphi_{5}\right)-$ regeneration in the desorber; 


$$
\varphi_{5}=\mathrm{L}\left(\mathrm{X}_{5}\right)=\left\{\mathrm{p}_{6} \mathrm{X}_{5} \mid \mathrm{X}_{5} \rightarrow \mathrm{p}_{6} \mathrm{p}_{7} \mathrm{p}_{8}\right\}=\left\{\mathrm{p}_{6} \mathrm{p}_{7} \mathrm{p}_{8}\right\},
$$

$r_{1}$ - verifies that the regenerated solution is present; $r_{2}$ - verifies that the regenerated solution from the bottom part of the desorber is present.

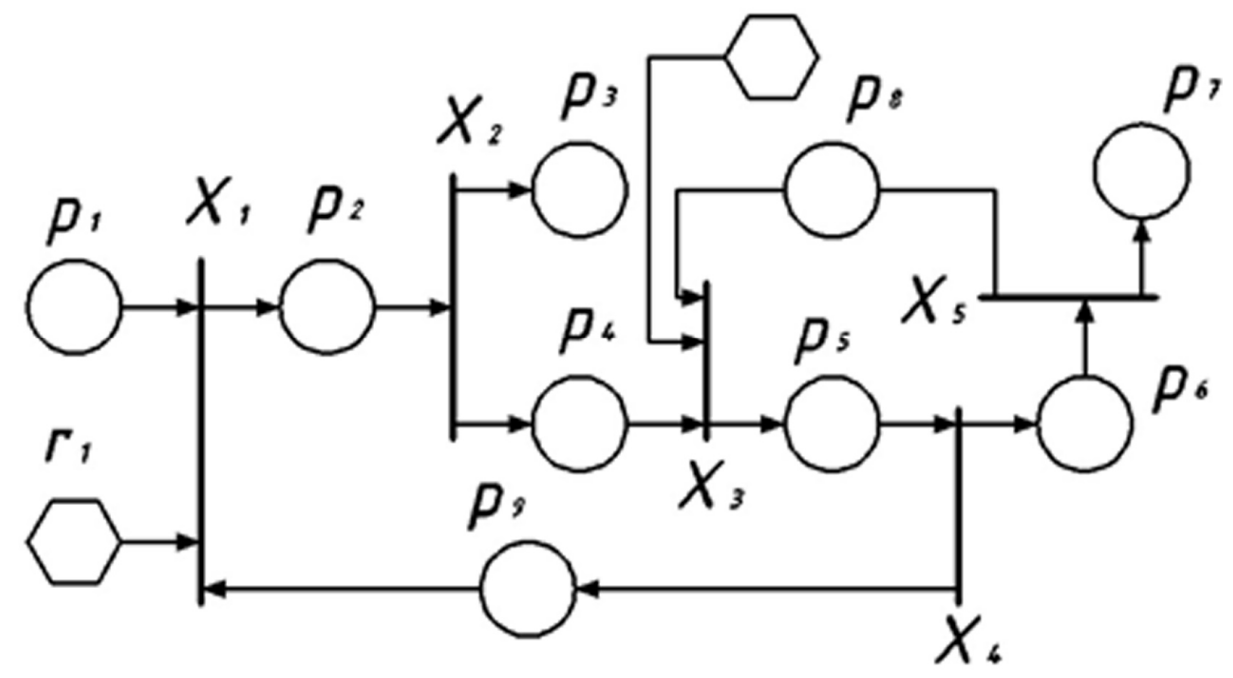

Fig. 5. Model of the carbon dioxide capture and storage system in terms of E-nets

For a realistic representation of the system for purification of gas emission from carbon dioxide, solid models of equipment and pipelines are built in several graphic editors based on the available technical documentation. Then, taking into account the planned design area and arrangement of the equipment, a three-dimensional image of the future operational line and technological process is created as shown in Fig. 6. Such an image allows to visually see the technical flaws in the theoretical planning phase and eliminate them.

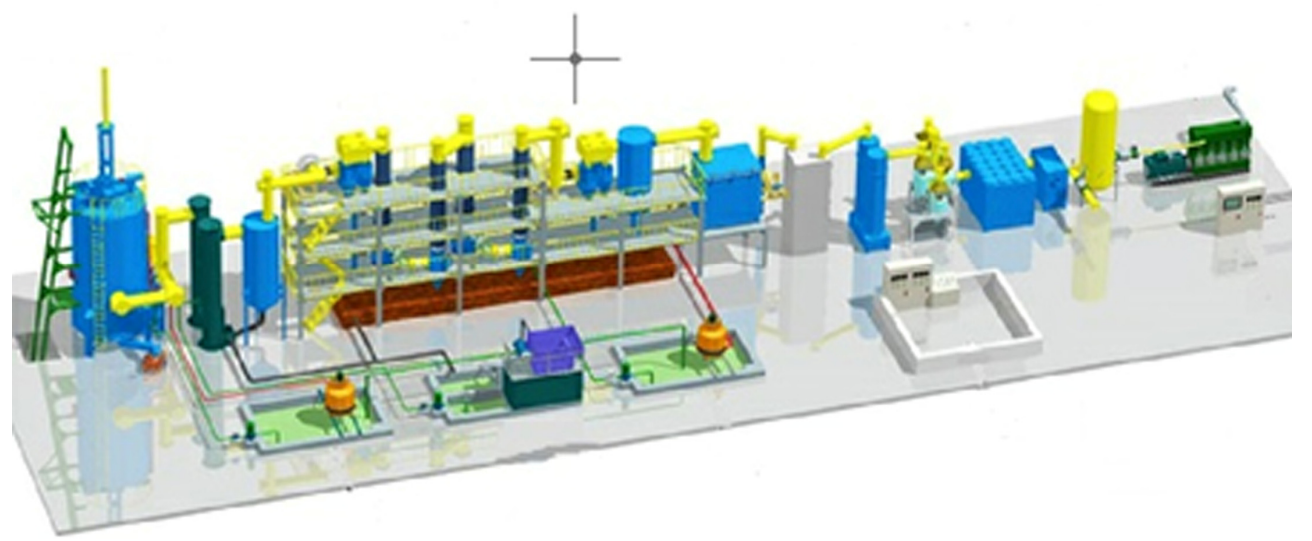

Fig. 6. Three-dimensional model of carbon dioxide capture and storage system.

Similarly, all modules of the subsystem for purification of gas emissions from a TPP can be analyzed. In particular, to calculate parameters of the module of the carbon dioxide capture system, the formula given below is used:

$$
\mathrm{M}_{\mathrm{CO}_{2}}=0,01 \cdot \mathrm{m} \cdot 3,664 \cdot \mathrm{C}^{\mathrm{p}} \cdot(1-0,01 \cdot \mathrm{q}),
$$


where $\mathrm{M}_{\mathrm{CO}_{2}}$ is the gross emission of carbon dioxide; $\mathrm{C}^{\mathrm{p}}$ is the carbon content in fuel, in $\%$; $\mathrm{q}$ is the loss of heat due to mechanical incompleteness of combustion of solid or liquid fuel, in $\%$.

The amount of materials (in tons) formed during the processing of 18,000 tons of coal: solid particles - 0.5837; Carbon dioxide - 105.4; Nitric oxide - 0.01684; Sulfur oxides 1.782; gold and silver $(<10 \%)-0.00001818$; Coagulant of aluminum sulfate - 2764; Uranium - 6.48; Thorium - 0.1163, Tantalum, Niobium, Cerium, Yttrium - 32.4; Titanium oxide - 170.5; Concentrate of Zirconium with Hafnium - 1494; Gallium - 0.22479; liquid glass 3161; Electricity (in Mwh) - 112540.06. The results of the model are shown in Fig. 7.

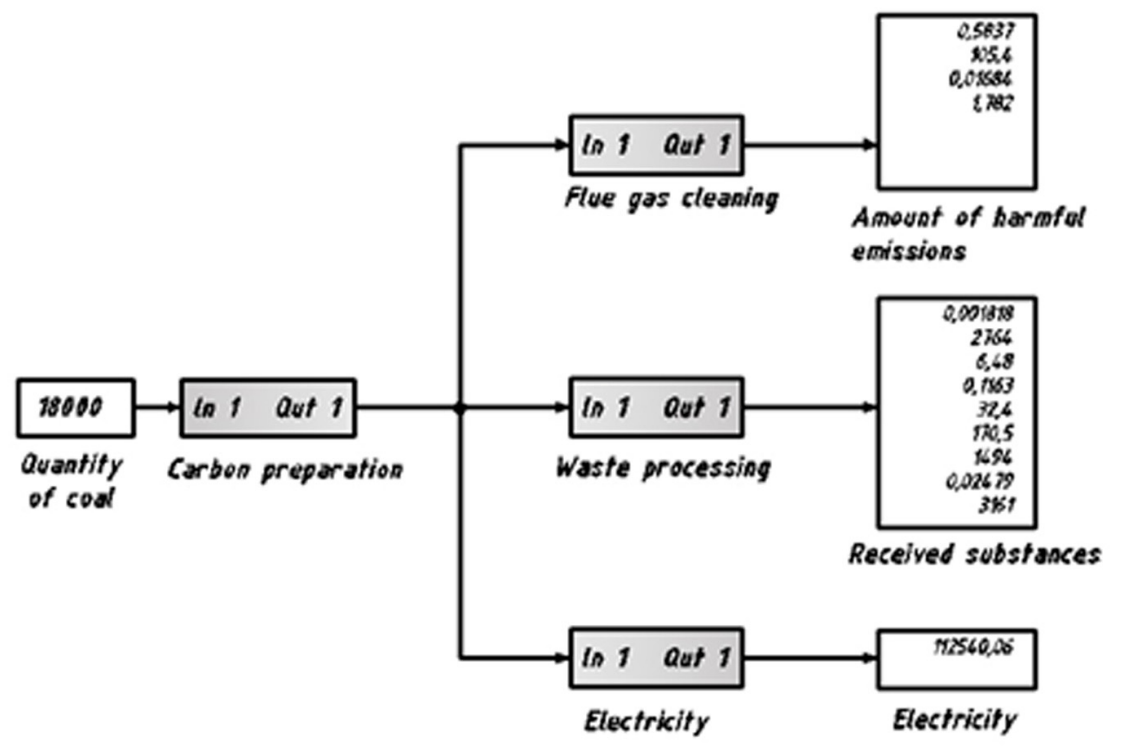

Fig. 7. The emission purification system of a TPP.

In conclusion, it should be noted that the resulting model can be further extended by adding necessary modules to its structure.

\section{Conclusions}

The problem of the application of mathematical and three-dimensional computer modeling tools for developing processes of fuel and energy complexes at the planning and design stage of a TPP is considered.

The solution of the problem of purification of gas emissions by means of mathematical and three-dimensional computer modeling is proposed. It allows to estimate the planned result, to choose the production technology, and also to ensure a high environmental effect of the planned waste treatment system. The results of processing the planned technological processes and the system for purifying gas emissions in terms of E-nets using mathematical modeling with the Simulink application are presented. A three-dimensional model of the gas emission purification system is constructed. This model allows to visualize the technological process and to compare it to theoretical calculations at the design stage of a TPP and, if needed, to make adjustments. 


\section{References}

1. Morad Behandish, Horea T., Computer-Aided Design, 70, 100 (2016)

2. Wenlan Ba, Ning Ren, Graphical Models, 82, 1 (2015)

3. N.G. Oliveira, J.L. Sotomayor, R.P. Torchelsen, C.T. Silva, L.D. João, Computers \& Graphics, 60, 119 (2016)

4. S.P. Bakhaeva, V.A. Gogolin, I.A. Ermakova, J. Min. Sci. 52:3, 454 (2016)

5. G.A. Kazunina, D.V. Alekseev, Adv. Mater. Res., 933, 62 (2014)

6. Hao Yang, Hui Zhang, Computers \& Graphics, 65, 45 (2017)

7. V.M. Zolotukhin, V.A. Gogolin, M.Yu. Yazevich, M.I. Baumgarten, A.V. Dyagileva, IOP Conf. Ser.: Earth Envir. Sci. 50:1, 012027 (2017)

8. I.A. Ermakova, N.N. Pirieva, Coal in the 21st Century: Mining, Processing and Safety, 90 (2016)

9. M. Tyulenev, A. Khoreshok, E. Garina, O. Litvin, Y. Litvin, E. A. Maliukhina, E3S Web Conf., 012035 (2017)

10. A. Khoreshok, L. Kantovich, V. Kuznetsov, E. Preis, D. Kuziev, E3S Web of Conferences, 15, 03004 (2017) 